\title{
Polymorphisms in PRKCDBP, a Transcriptional Target of TNF- $\alpha$, Are Associated With Inflammatory Bowel Disease in Korean
}

\author{
Jung-Wook Kim ${ }^{1}$, Chang Kyun Lee ${ }^{1}$, Hyo Jong Kim ${ }^{1}$, Jae-Jun Shim ${ }^{1}$, Jae Young Jang ${ }^{1}$, Seok Ho Dong ${ }^{1}$, \\ Byung-Ho Kim ${ }^{1}$, Young Woon Chang ${ }^{1}$, Sung-Gil Chi ${ }^{2}$ \\ Division of Gastroenterology, Department of Internal Medicine, Kyung Hee University School of Medicine', School of Life Sciences and \\ Biotechnology, Korea University², Seoul, Korea
}

\begin{abstract}
Background/Aims: Emerging data indicate that polymorphic sequence variations in the tumor necrosis factor alpha (TNF- $\alpha)$ gene may affect its production, and be associated with the risk of inflammatory bowel disease (IBD). PRKCDBP is a putative tumor suppressor gene and a transcriptional target of TNF- $\alpha$. The aim of this case-control study is to explore the possible association of single nucleotide polymorphisms (SNPs) in PRKCDBP with the development of IBD in Koreans. Methods: Genotyping analysis of four SNPs of PRKCDBP [rs35301211 (G210A), rs11544766 (G237C), rs12294600 (C797T), and rs1051992 (T507C)] was performed on 170 ulcerative colitis (UC),131 Crohn's disease (CD) patients, and 100 unrelated healthy controls using polymerase chain reaction and restriction fragment length polymorphism. Results: Heterozygous configuration of three SNPs (G210A, G237C, and C797T) was very rare in both patients and healthy controls. However, allele frequencies of the T507C SNP showed a significant difference between UC patients and controls $(P=0.037)$. The CC genotype of the T507C SNP was identified in $46.6 \%$ (61 of 131) of CD and 49.4\% (84 of 170) of UC patients, but only in 33.0\% (33 of 100) of healthy controls. Furthermore, CC homozygosity was more prevalent than TC heterozygosity in both CD and UC patients versus controls $(P=0.016$; genderadjusted odds ratio [aOR], 2.16; 95\% confidence interval [CI], 1.16-4.04 and $P=0.009$; aOR, 2.09; 95\% CI, 1.193.64; respectively) Conclusions: Our results suggest that the T507C SNP in PRKCDBP, a TNF- $\alpha$-inducible gene, might be associated with susceptibility to IBD (particularly UC) development in Koreans. (Intest Res 2015;13:242-249)
\end{abstract}

Key Words: PRKCDBP; Polymorphisms, single nucleotide; Crohn disease; Colitis, ulcerative

\section{INTRODUCTION}

Crohn's disease (CD) and UC are the two main forms of chronic IBD, characterized by remitting and relapsing inflammation of the bowel. As with other complex diseases, both genetic susceptibility and environmental factors appear

Received December 16, 2014. Revised March 22, 2015

Accepted April 10, 2015

Correspondence to Chang Kyun Lee, Division of Gastroenterology, Department of Internal Medicine, Kyung Hee University School of Medicine, 23 Kyungheedae-ro, Dongdaemun-gu, Seoul 130-872, Korea. Tel: +82-2958-8258, Fax: +82-2-968-1848, E-mail: gidrlee@paran.com

Financial support: None. Conflict of interest: None. to be involved in the pathogenesis of IBD. Accumulating evidence suggests that IBD results from an inappropriate and continuing inflammatory response to intestinal microbes in genetically susceptible hosts. ${ }^{1}$

To date, genome-wide association studies (GWASs) and meta-analyses of IBD have identified $140 \mathrm{CD}$-susceptibility loci and 47 UC-susceptibility loci, including 28 that are shared between CD and UC. ${ }^{2-6}$ Of these, the tumor necrosis factor (TNF) superfamily member 15 (TNFSF15) variant contributes to CD susceptibility in European, ${ }^{7}$ Japanese, ${ }^{7,8}$ and Korean populations. ${ }^{9}$ TNFSF15 is abundantly expressed, primarily in endothelial cells, ${ }^{10}$ and is a ligand for receptors such as the TNF receptor superfamily member 25

\footnotetext{
(c) Copyright 2015. Korean Association for the Study of Intestinal Diseases. All rights reserved.

This is an Open Access article distributed under the terms of the Creative Commons Attribution Non-Commercial License (http://creativecommons.org/licenses/by-nc/4.0)

which permits unrestricted non-commercial use, distribution, and reproduction in any medium, provided the original work is properly cited.
} 
(TNFRSF25). ${ }^{11}$ Its expression has been shown to be highly inducible by $\mathrm{TNF} \alpha$ and interleukin-1- $\alpha$. It also activates nuclear factor (NF)- $\mathrm{KB}$ and induces apoptosis in TNFRSF25expressing cell lines. ${ }^{11}$ Because TNF- $\alpha$ is a proinflammatory cytokine that plays a key role in the immunopathogenesis of IBD, it seems reasonable to suspect that TNFSF15 genotypes may play an important role in the pathogenesis of $\mathrm{CD}$.

Similarly, although it has not been investigated in IBD, protein kinase $\mathrm{C}$, delta-binding protein (PRKCDBP, also known as Cavin3/hSRBC) is a novel candidate gene in IBD. Originally, Izumi et al. ${ }^{12}$ isolated a protein kinase C-binding protein, the expression of which was induced by serum starvation. $P R K C D B P$ was recently identified as a proapoptotic tumor suppressor, located at chromosomal region $11 \mathrm{p} 15.5$ p15.4. ${ }^{13,14}$ We reported that $P R K C D B P$ induces cell cycle arrest and apoptotic cell death by enhancing the protein stability of p53. ${ }^{14}$ Consistent with these findings, loss or reduction of PRKCDBP expression due to aberrant promoter CpG site hypermethylation has been observed in many malignancies, including colorectal cancer. ${ }^{13-15}$ Interestingly, we found that $P R K C D B P$ transcription is activated directly by NF- $\kappa \mathrm{B}$ signaling in response to TNF- $\alpha$, which plays a key role in colonic inflammation and tumorigenesis, and its inactivation contributes to tumor growth and increased resistance to TNF- $\alpha$ induced apoptosis. ${ }^{16}$ Moreover, our recent study demonstrated that colonic mucosal expression of PRKCDBP correlates strongly with TNF $\alpha$ expression in UC patients. ${ }^{17}$ These observations suggest that $P R K C D B P$ may play a role in colonic inflammation, and its dysregulation may be implicated in the pathogenesis of IBD.

The PRKCDBP gene has more than 30 intraexonic single nucleotide polymorphisms (SNPs) leading to amino acid substitutions. To our knowledge, only one reported study has evaluated the contribution of the PRKCDBP genotype to endometrial carcinogenesis. ${ }^{18}$ To explore the possible association of SNPs in PRKCDBP with the development of IBD, we conducted a case-control study for four representative intraexonic SNPs (rs35301211, rs11544766, rs12294600, and rs 1051992) based on their reported heterozygosity rates in Asian populations.

\section{METHODS}

\section{Study Subjects}

In total, 170 UC patients, 131 CD patients, and 100 unrelated healthy controls were included in this case-control study from May 1998 to January 2008. Blood samples were obtained from all subjects in EDTA-coated tubes. All subjects were Koreans, unrelated, over 18 years of age, and with no family history of IBD. Clinical data were obtained by detailed reviews of case records. All patients were diagnosed at the Department of Gastroenterology, Kyung Hee University Hospital, Seoul, Korea, on the basis of clinical, radiological, endoscopic, and histopathological findings according to conventional criteria. Disease phenotypes were defined following the Vienna classification ${ }^{19}$ for disease location and type of disease (penetrating, structuring, or inflammatory) in CD. UC patients were classified according to the location and extent of the inflammatory lesions (proctitis, leftsided colitis, or pancolitis). Healthy controls were volunteers without a family history of IBD. They had no gastrointestinal symptoms, took no regular medication, and had no other diseases. The study was conducted in accordance with the Declaration of Helsinki, and all subjects provided informed consent

\section{SNPs}

The human genome database (http://www.ncbi.nlm.nih. gov/sites/entrez) shows that the PRKCDBP gene has more than 100 intragenic SNPs, 36 of which are missense changes causing to amino acid substitutions. We chose four representative intraexonic SNPs, rs35301211 (G210A), rs11544766 (G237C), rs12294600 (C797T), and rs1051992 (T507C) based on their reported heterozygosity rates in Asians and the National Center for Biotechnology Information database (http://www.ncbi.nlm.nih.gov/projects/SNP/snp_ref. cgi?geneId $=112464){ }^{20}$

\section{Extraction of Genomic DNA}

Genomic DNA was extracted from blood lymphocytes by standard methods. Briefly, blood lymphocytes were incubated with an extraction buffer containing $1 \mathrm{M} \mathrm{KCl}, 1 \mathrm{M} \mathrm{MgCl}_{2}$, $1 \%$ Tween $20,1 \%$ Nonidet P-40, and $2.5 \mu \mathrm{m} / \mathrm{mL}$ proteinase $\mathrm{K}$, at $55^{\circ} \mathrm{C}$ for 1 hour. Proteinase $\mathrm{K}$ was inactivated by heating at $95^{\circ} \mathrm{C}$ for 10 minutes and the supernatant containing genomic DNA was stored at $-20^{\circ} \mathrm{C}$. The concentration of the extracted DNA was determined by spectrophotometric measurements.

\section{PCR Amplification of Gene Regions Including SNPs}

To determine the genotypes of the SNPs in patients and healthy controls, the gene regions including each SNP 
were separately amplified by PCR using primers SRBC2101 and SRBC210-2 for G210A, SRBC237-1 and SRBC2372 for G237C, SRBC507-1 and SRBC507-2 for T507C, and SRBC797-1 and SRBC797-2 for C797T (Table 1). For restriction enzyme digestion assays of the PCR products, one nucleotide around the 3' end of SRBC210-2 and SRBC7971 was replaced with a non-complementary nucleotide to generate the recognition site for SphI and BtsI. PCR was performed with $200 \mathrm{ng}$ of genomic DNA as a template for 38 cycles of $95^{\circ} \mathrm{C}$ for 1 minute, $60^{\circ} \mathrm{C}$ for 0.5 minute, and $72^{\circ} \mathrm{C}$ for 1 minute in $1.5 \mathrm{mM} \mathrm{MgCl}_{2}$-containing reaction buffer (PCR buffer II; Perkin Elmer-Cetus, Norwalk, CT, USA). PCR products $(10 \mu \mathrm{L})$ were resolved on $2 \%$ agarose gels, stained with ethidium bromide $(0.5 \mu \mathrm{g} / \mathrm{mL}$ in $1 \mathrm{X}$ TBE$)$, visualized using ultraviolet light, and photographed.

\section{RFLP Analysis}

PCR products $(10 \mu \mathrm{L})$ were digested with the restriction enzymes $S p h \mathrm{I}$ (GCATG $\downarrow$ C, SNP is underlined) for G210A, DdeI (C $\downarrow$ TNA $\underline{\text { }}$ ) for G237C, BtsI ( $\downarrow$ CACTG $\underline{\text { C }}$ ) for C797T, and $P_{v u I I}(\mathrm{CAG} \downarrow \mathrm{CTG})$ for $\mathrm{T} 507 \mathrm{C}$ overnight at $37^{\circ} \mathrm{C}$, according to the manufacturers' recommendations. The PCR products digested by each restriction enzyme displayed two fragments as follows: $S p h$ I digestion of a G allele (184 bp and 24 bp), DdeI digestion of a G allele (199 bp and 140 bp), Bts I digestion of a $\mathrm{C}$ allele (209 bp and $22 \mathrm{bp}$ ), and $P_{v u I I}$ digestion of a $\mathrm{T}$ allele (167 bp and $32 \mathrm{bp}$ ). The restriction digest products were analyzed using electrophoresis on a $2.0 \%$ agarose gel.

\section{Single-Strand Conformation Polymorphism (SSCP) Analysis}

To confirm the results of the PCR-RFLP assays, SSCP analysis was performed using the same PCR products. Briefly, PCR products $(20 \mu \mathrm{L})$ were mixed with $5 \mu \mathrm{L}$ of 0.5 $\mathrm{N} \mathrm{NaOH}, 10 \mathrm{mM}$ EDTA, and $10 \mu \mathrm{L}$ of denaturing loading buffer (95\% formamide, $20 \mathrm{mM}$ EDTA, 0.05\% bromophenol blue, and $0.05 \%$ xylene cyanol). After heating at $95^{\circ} \mathrm{C}$ for 5 minutes, samples were loaded rapidly into wells pre-cooled to $4^{\circ} \mathrm{C}$ and run simultaneously on two $8 \%$ non-denaturing polyacrylamide gels with and without $10 \%$ glycerol. These two gels were then run at $18-20^{\circ} \mathrm{C}$ and this was repeated at $6-10^{\circ} \mathrm{C}$ in a buffer-jacketed gel apparatus (DGGE-II; Aladin Enterprises, Inc., San Francisco, CA, USA). Following a 4-hour run at 460 volts, the gels were stained with ethidium bromide and photographed under ultraviolet light. The PCRRFLP assay and SSCP analysis yield identical results.

\section{DNA Sequencing Analysis of PCR Products}

PCR products showing different SSCP patterns were subjected to DNA sequencing analysis to verify the polymorphisms present. Cloning of the PCR products for sequencing analysis was performed using the TA cloning kit (Invitrogen, San Diego, CA, USA), and at least three clones showing identical SSCP abnormalities were selected and sequenced. Sequencing was performed in both directions to confirm the findings.

Table 1. Oligonucleotide Primers Used for Genomic PCR of Four Intraexonic Single Nucleotide Polymorphisms (SNPs) in PRKCDBP (All sequences are listed 5 ' to $\left.3^{\prime}\right)$

\begin{tabular}{cllc}
\hline Primers & SNPs & \multicolumn{1}{c}{ Sequences } & Orientation \\
\hline SRBC210-1 & G210A & GATCATGAGGGAGAGTGCGTGGAGC & Sense \\
SRBC210-2 & & ACTCAGAGCGGCCAGGCCGCTCTGCAT & Antisense \\
SRBC237-1 & G237C & ATGAGGGAGAGTGCGTGGAGCGGG & Sense \\
SRBC237-2 & & GTGGTGGCCTCCAGCCGCTGCACCT & Antisense \\
SRBC507-1 & T507C & CGGCGGACCAGTCCGAGCTG & Sense \\
SRBC507-2 & & CCAAGGCGAGGCGGCTGACC & Antisense \\
SRBC797-1 & C797T & AGACCTGGGGCTGCCGAAGAAGCACT & Sense \\
SRBC797-2 & & CAGGTGTGAGTGACTGCACCTCTTCA & Antisense \\
\hline
\end{tabular}

Italic indicates non-complementary nucleotide included to generate restriction enzyme recognition site (underlined).

$P R K C D B P$, protein kinase $\mathrm{C}$, delta binding protein. 


\section{Statistical Analysis}

The chi-square test was used to assess any deviation from Hardy-Weinberg equilibrium of the PRKCDBP polymorphisms in the patients and controls. The genotype frequencies were expressed as percentages of chromosomes containing a variant allele. The association between IBD patients and healthy controls was examined by comparing allele and genotype frequencies in different subject groups using a chisquare test. Allelic frequencies were compared with patients and controls using logistic regression analysis to calculate age- and gender-adjusted OR (aOR) and $95 \%$ CIs. A $P$-value of less than 0.05 was considered to indicate statistical significance. The strength of association was estimated by the OR. Data were analyzed using SPSS (ver. 13.0 for Windows; SPSS, Inc., Chicago, IL, USA).

\section{RESULTS}

\section{Characteristics of the Study Population}

Demographic and clinical characteristics of the study subjects are summarized in Table 2. In total, 170 UC patients, 131 CD patients, and 100 healthy controls were enrolled. CD patients were younger than healthy controls $(P<0.001)$ and UC patients were older than healthy controls $(P=0.003)$. The male-to-female ratio of CD patients was significantly higher than that of the other groups. According to extent of disease, ileocolic disease in CD (66.4\%) and proctitis in UC (38.2\%) were most common. The genotype frequencies in UC and CD patients and controls conformed to the Hardy-Weinberg equilibrium $(P>0.05$, data not shown).

\section{Infrequent Sequence Variation at G210A, G237C, and C797T in a Korean Population}

PCR-RFLP analysis of G210A SNP displayed only digested PCR fragments (G allele) from all 170 UC, 131 CD patients, and 100 healthy individuals, indicating no sequence variation at this site (Fig. 1A). Genotype analysis of the G237C SNP detected a GT heterozygote configuration in only one CD case, and all other patients and healthy individuals showed the GG homozygote configuration (Fig. 1B). Similarly, genotype analysis of the C797T SNP identified a CT heterozygote configuration from two $\mathrm{CD}$, two UC patients, and two healthy controls. All other patients and healthy controls had the CC genotype (Fig. 1C).

Table 2. Clinical Characteristics of the Study Subjects

\begin{tabular}{|c|c|c|c|}
\hline Variables & $C D(n=131)$ & UC $(n=170)$ & $\mathrm{HC}(n=100)$ \\
\hline \multicolumn{4}{|l|}{ Gender } \\
\hline Male & $99(75.6)^{*}$ & $86(50.6)$ & $58(58.0)$ \\
\hline Female & $32(24.4)$ & $84(49.4)$ & $42(42.0)$ \\
\hline Age (yr) & $30.4 \pm 11.2^{+}$ & $44.0 \pm 14.4^{\dagger}$ & $38.5 \pm 15.3$ \\
\hline Age at diagnosis (yr) & $24.5 \pm 9.4$ & $38.5 \pm 13.8$ & \\
\hline Mean duration of disease at sampling (mo) & $62.1 \pm 61.5$ & $71.9 \pm 62.9$ & \\
\hline Surgery & $72(55.0)$ & $31(18.2)$ & \\
\hline \multirow[t]{3}{*}{ Disease extent } & Terminal ileum: 35 & Proctitis: 65 & \\
\hline & Colon: 13 & Left-sided: 63 & \\
\hline & Ileocolon: 83 & Pancolitis: 42 & \\
\hline \multicolumn{4}{|l|}{ Disease behavior } \\
\hline Inflammatory & $64(48.9)$ & & \\
\hline Stricturing & $49(37.4)$ & & \\
\hline Penetrating & $18(13.7)$ & & \\
\hline Perianal disease & $40(30.5)$ & & \\
\hline
\end{tabular}

Values are presented as $n(\%)$ or mean \pm SD.

${ }^{*} P$-value was 0.005 , it was derived from $\chi^{2}$ test between $C D$ and $H C$.

${ }^{\dagger} P$-value was $<0.001$, it was derived from independent $t$-test between $\mathrm{CD}$ and $\mathrm{HC}$.

${ }^{\ddagger} P$-value was 0.003 , it was derived from independent $t$-test between $\mathrm{UC}$ and $\mathrm{HC}$.

$\mathrm{HC}$, healthy control. 


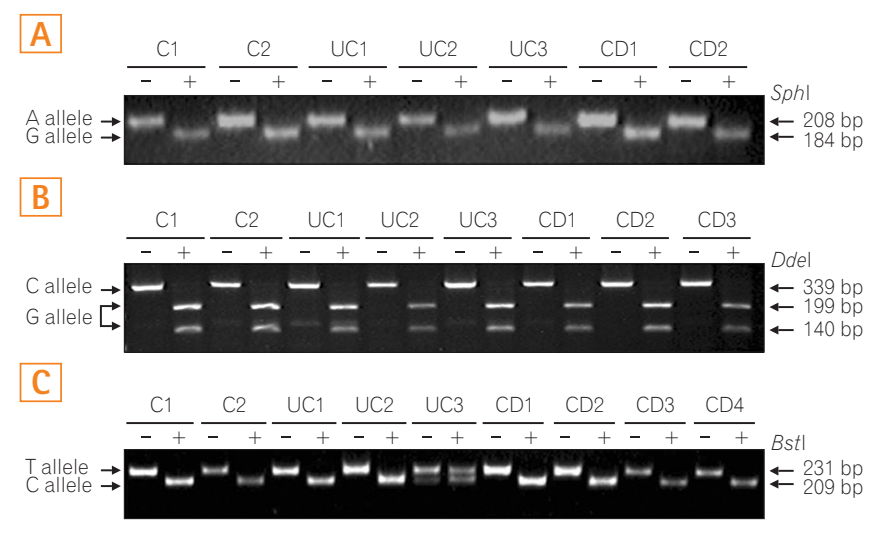

Fig. 1. Representative genotyping of single nucleotide polymorphisms (SNPs) by PCR-RFLP. (A) Genotype analysis of the G201A SNP. The 208bp fragment is the undigested PCR product of the A allele. Two fragments of 184 and 24 bp lengths result from Sphl digestion of the $G$ allele. Only the 184-bp fragment was detectable on a $2 \%$ agarose gel. (B) Genotype analysis of the G237C SNP. The 339-bp fragment is the undigested PCR product of the C allele. Two fragments of 199 and $140 \mathrm{bp}$ lengths result from Ddel digestion of the $\mathrm{G}$ allele. (C) Genotype analysis of the C797T SNP. The 231-bp fragment is the undigested PCR product of the T allele. Two fragments of 209 and 22 bp lengths result from Btsl digestion of the C allele. Only the 209-bp fragment was detected.

\section{Association Between T507C SNP and IBD Development}

In contrast to the G210A, G237C, and C797T SNPs, the T507C SNP (5'CAG $\downarrow$ CTG-3', PvuII digestion) showed high heterozygosity in both the patients and control groups (Fig. 2 ). The allele frequencies of $\mathrm{T} 507 \mathrm{C}$ were found to have differing distributions in IBD patients and controls. The frequencies of the T (Leu) and C (Pro) allele were $41 \%$ and $59 \%$ in healthy controls, but $35.5 \%$ and $64.5 \%$ in CD and $32.1 \%$ and $67.9 \%$ in UC patients. With respect to allele frequencies of the T507C SNP, patients with UC showed significant differences from controls under logistic regression analysis $(P=0.037)$ (Table 3), while CD patients did not $(P=0.194)$ (Table 4). The genotype frequencies also had different distributions in patients and controls. The TT genotype frequencies were $17.6 \%$ in CD, $13.5 \%$ in UC, and $15.0 \%$ in healthy controls, while CC genotype frequencies were $46.6 \%$ in CD, $49.4 \%$ in UC, and $33.0 \%$ in healthy controls. Patients with $\mathrm{CD}$ and UC were more likely to be $507 \mathrm{CC}$ homozygous $(P=0.016$; aOR, 2.16; 95\% CI, 1.16-4.04; $P=0.009$; aOR, 2.09; 95\% CI, 1.19-3.64; respectively) rather than TC heterozygous compared with healthy controls (Tables 3, 4).
A SRBC507-1 gcccggcgga ccagtccgag ctgggcccag aocagctgga ggccgaagtt ggagagagct cggacgagga gccggtggag tccagggccc agcggctgcg gcgcaccgga ttgcagaagg tacagagcct ccgaagggcc ctttcgggcc ggaaaggccc tgcagcgcca ccgcccaccc cggtcaagcc gcctcgcctt gggcctggcc

B

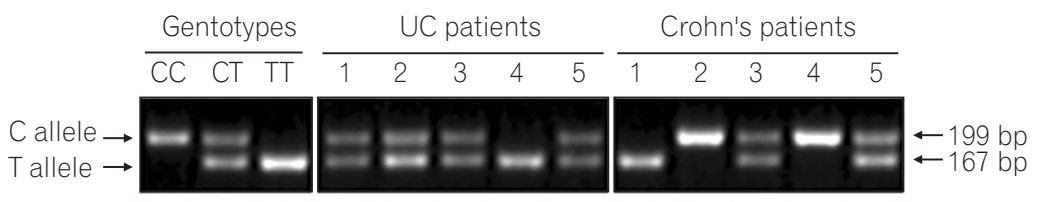

Fig. 2. The $T 507 \mathrm{C}$ single nucleotide polymorphisms (SNP). (A) The genomic sequence region flanking the T507C single nucleotide polymorphism (SNP) site. It was amplified by PCR using primers SRBC5071 and SRBC507-2 (underlined). The SNP site is denoted by bold text and a box. (B) Representative genotyping of the PRKCDBP rs 1051992 (T507C) SNP. The 199 bp fragment is the undigested product of the $C$ allele. Fragments of 167 and 32 bp lengths result from Pvull digestion of the T allele. Only the $167 \mathrm{bp}$ fragment of the digested PCR products was detectable on a $2 \%$ agarose gel. 


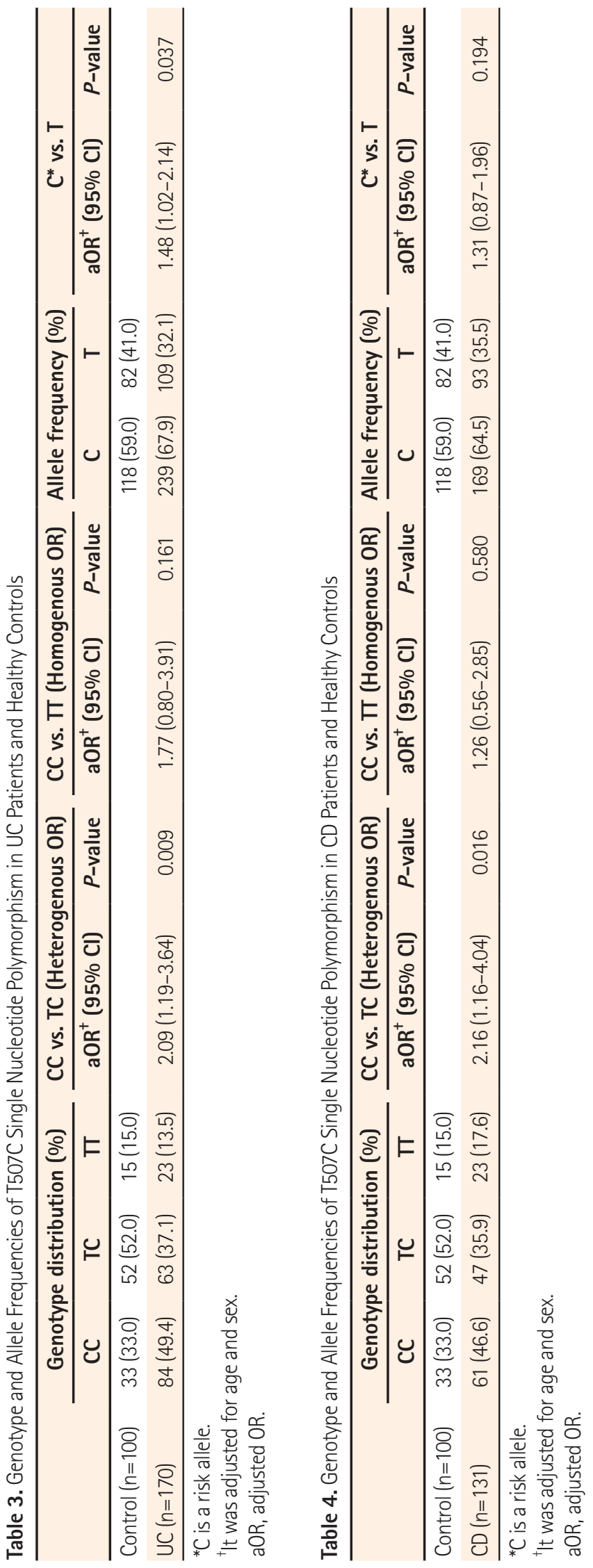

\section{DISCUSSION}

The present study represents an evaluation of $P R C K D B P$ polymorphisms as potential factors in IBD susceptibility. $P R K C D B P$ is a putative tumor suppressor that is altered in several human malignancies. ${ }^{13-15}$ PRKCDBP is a transcriptional target of TNF- $\alpha-\mathrm{NF} \kappa \mathrm{B}$ signaling, which plays a key role in TNF- $\alpha$-induced apoptosis, ${ }^{16}$ and is frequently lost or downregulated in patients with human colorectal cancer. ${ }^{16}$ TNF- $\alpha$ is an important pro-inflammatory cytokine involved not only in inflammatory responses, but also apoptosis, cell proliferation, and differentiation. ${ }^{21,22}$ Thus, it is not surprising that genetic variation in the TNF- $\alpha$ gene contributes to susceptibility to IBD. Several studies have reported associations between the TNF- $\alpha$ gene and the development of IBD. SNPs in the promoters of the $T N F-\alpha^{23}$ and $T N F S F 1^{7,9,24}$ genes are involved in genetic predispositions in diverse ethnic groups. Although TNF- $\alpha$ has been implicated in the pathogenesis of IBD, the role of PRKCDBP has not been recognized in IBD previously. Our previous report is the only to implicate PRKCDBP in IBD. ${ }^{17}$ In this previous study, we demonstrated that, PRKCDBP expression is tightly controlled by TNF- $\alpha$ in patients with moderate to severe UC. ${ }^{17}$ Along with our previous result, considering that $P R K C D B P$ is a proapoptotic tumor suppressor and a transcriptional target of TNF- $\alpha$, it seems probable that these missense SNPs could generate variant protein products, with varying levels of biological activity. Nevertheless, the association between the SNPs of $P R K C D B P$ and susceptibility to IBD has not yet been determined.

In the present study, four representative intraexonic SNPs (G210A, G237C, T507C, and C797T), which lead to amino acid substitutions, were chosen based on their heterozygosity rates in Asian populations, and we investigated their possible association with susceptibility to IBD. Extremely low heterozygote configurations at three SNP sites (G210A, $\mathrm{G} 237 \mathrm{C}$, and $\mathrm{C} 797 \mathrm{~T}$ ) were seen. The major genotype (GG, GG, and CC, respectively) of these SNPs was found in more than $98 \%$ of all groups. Thus, minor variants of these three SNPs in the PRKCDBP gene are very rare in Korean populations. This also indicates that G210A, G237C, and C797T SNPs are not likely associated with susceptibility to IBD, and therefore not useful for the identification of groups at high risk of IBD in Korea. However, this study demonstrated that the genotype frequencies of the T507C SNP of the PRKCDBP gene showed different distributions in IBD patients and healthy controls. The CC genotype frequency was significantly higher in IBD patients than in healthy controls, but 
TT and CT genotypes were not different between the two groups. Further, the frequencies of the $\mathrm{T}$ (Leu) and $\mathrm{C}$ (Pro) alleles were $41 \%$ and $59 \%$ in healthy controls, but UC patients showed a significantly higher frequency of the $\mathrm{C}$ allele (67.9\%, $P=0.037$ ). In CD patients, the frequency of the $\mathrm{C}$ allele was also higher than in healthy controls, but this was not statistically significant $(64.5 \%, P=0.194)$. The finding of a considerable frequency of the $\mathrm{C}$ allele in healthy controls demonstrated a weak association between IBD and this SNP of the PRKCDBP gene. Furthermore, it means that PRKCDBP may partially contribute to the pathogenesis of IBD having extensive genetic heterogeneity.

GWASs have identified approximately 163 independent loci for IBD, 92 of which were further confirmed and 71 newly established in the recent International IBD Genetics Consortium "ImmunoChip" study. ${ }^{6}$ Recent Korean studies have validated GWASs in Caucasian populations, and found novel susceptibility genes for IBD. ${ }^{4,5}$ Products of these candidate genes mediate various cellular functions, including microbial recognition, ${ }^{25}$ lymphocyte activation ${ }^{26}$ cytokine signaling ${ }^{20}$ metabolism, ${ }^{27}$ and epithelial barrier function. ${ }^{28}$ Thus, mutations in these genes may be associated with the development of IBD. However, specific functional variations have been demonstrated in only a small number of genes, including NOD2, IL23R, ATG16L1, IRGM, and PTPN222. ${ }^{29,30}$ Our present study adds a novel candidate gene, $P R K C D B P$, to the list of genes associated with the development of IBD. The possible mechanism of the association between PRKCDBP and susceptibility to IBD has not been established. Based on our previous study, ${ }^{17}$ we speculated that PRKCDBP may play a role in the inflammatory processes controlled by TNF- $\alpha$ in IBD.

Our study has some limitations. First, a functional analysis of $P R K C D B P$ was not performed, and the exact functional consequences of $P R K C D B P$ polymorphisms remain to be elucidated. Second, we did not analyze the clinical characteristics of the patients. Therefore, we could not investigate the relationship between genotypes and phenotypes. Furthermore, we could not exclude the possibility that age imbalances among groups may affect the results because of a lack of clinical data. Third, this was a single-center study conducted in a tertiary teaching hospital in Korea. Therefore, it is not possible to determine whether our results true for other populations. Finally, the number of specimens tested in this study was small. Thus, to confirm the association between PRKCDBP polymorphisms and the development of IBD, a replication of the association study in other populations with a larger number of patients is needed.
Despite these limitations, the results raise the possibility that the T507C SNP of PRKCDBP, a TNF- $\alpha$-inducible gene with proapoptotic activity, might help to identify patient subgroups at high risk of IBD development. Collectively, these findings suggest that $P R K C D B P$ genetic variants might be associated with susceptibility to IBD development, especially in UC. To our knowledge, this is the first study of PRKCDBP polymorphisms in IBD reported. It is possible that the TNF $\alpha /$ PRKCDBP signaling pathway may play a role in IBD pathogenesis, based on this result and previous reports. ${ }^{1,14,17,18}$ However, the involvement of PRKCDBP in the pathogenesis of IBD has not been investigated. Thus, further studies at the molecular level and in the clinical setting are needed to explore this possibility in IBD.

\section{REFERENCES}

1. Abraham C, Cho JH. Inflammatory bowel disease. N Engl J Med 2009;361:2066-2078.

2. Anderson CA, Boucher G, Lees CW, et al. Meta-analysis identifies 29 additional ulcerative colitis risk loci, increasing the number of confirmed associations to 47. Nat Genet 2011;43:246252.

3. Franke A, McGovern DP, Barrett JC, et al. Genome-wide metaanalysis increases to 71 the number of confirmed Crohn's disease susceptibility loci. Nat Genet 2010;42:1118-1125.

4. Yang SK, Hong M, Zhao W, et al. Genome-wide association study of Crohn's disease in Koreans revealed three new susceptibility loci and common attributes of genetic susceptibility across ethnic populations. Gut 2014;63:80-87.

5. Yang SK, Hong M, Zhao W, et al. Genome-wide association study of ulcerative colitis in Koreans suggests extensive overlapping of genetic susceptibility with Caucasians. Inflamm Bowel Dis 2013;19:954-966.

6. Jostins L, Ripke S, Weersma RK, et al. Host-microbe interactions have shaped the genetic architecture of inflammatory bowel disease. Nature 2012;491:119-124.

7. Yamazaki K, McGovern D, Ragoussis J, et al. Single nucleotide polymorphisms in TNFSF15 confer susceptibility to Crohn's disease. Hum Mol Genet 2005;14:3499-3506.

8. Kakuta Y, Kinouchi Y, Negoro K, Takahashi S, Shimosegawa T. Association study of TNFSF15 polymorphisms in Japanese patients with inflammatory bowel disease. Gut 2006;55:1527-1528.

9. Yang SK, Lim J, Chang HS, et al. Association of TNFSF15 with Crohn's disease in Koreans. Am J Gastroenterol 2008;103:14371442. 
10. Yue TL, Ni J, Romanic AM, et al. TL1, a novel tumor necrosis factor-like cytokine, induces apoptosis in endothelial cells. Involvement of activation of stress protein kinases (stress-activated protein kinase and p38 mitogen-activated protein kinase) and caspase-3-like protease. J Biol Chem 1999;274:1479-1486.

11. Migone TS, Zhang J, Luo X, et al. TL1A is a TNF-like ligand for DR3 and TR6/DcR3 and functions as a T cell costimulator. Immunity 2002;16:479-492.

12. Izumi Y, Hirai S, Tamai Y, Fujise-Matsuoka A, Nishimura Y, Ohno S. A protein kinase Cdelta-binding protein SRBC whose expression is induced by serum starvation. J Biol Chem 1997;272:7381-7389.

13. Xu XL, Wu LC, Du F, et al. Inactivation of human $S R B C$, located within the 11 p15.5-p15.4 tumor suppressor region, in breast and lung cancers. Cancer Res 2001;61:7943-7949.

14. Lee JH, Byun DS, Lee MG, et al. Frequent epigenetic inactivation of $h S R B C$ in gastric cancer and its implication in attenuated p53 response to stresses. Int J Cancer 2008;122:1573-1584.

15. Zöchbauer-Müller S, Fong KM, Geradts J, et al. Expression of the candidate tumor suppressor gene $h S R B C$ is frequently lost in primary lung cancers with and without DNA methylation. Oncogene 2005;24:6249-6255.

16. Lee JH, Kang MJ, Han HY, et al. Epigenetic alteration of $P R K C D B P$ in colorectal cancers and its implication in tumor cell resistance to TNF $\alpha$-induced apoptosis. Clin Cancer Res 2011;17:7551-7562.

17. Kim JW, Kim HJ, Lee CK, et al. Elevation of PRKCDBP, a novel transcriptional target of TNF- $\alpha$, and its downregulation by infliximab in patients with ulcerative colitis. Dig Dis Sci 2014; 59:2947-2957.

18. Tong SY, Lee JM, Ki KD, Seol HJ, Choi YJ, Lee SK. Genetic polymorphism of PRKCDBP is associated with an increased risk of endometrial cancer. Cancer Invest 2012;30:642-645.

19. Gasche C, Scholmerich J, Brynskov J, et al. A simple classification of Crohn's disease: report of the Working Party for the World Congresses of Gastroenterology, Vienna 1998. Inflamm Bowel Dis 2000;6:8-15.
20. Derynck R, Zhang YE. Smad-dependent and Smad-independent pathways in TGF- $\beta$ family signalling. Nature 2003;425:577584.

21. Gaur U, Aggarwal BB. Regulation of proliferation, survival and apoptosis by members of the TNF superfamily. Biochem Pharmacol 2003;66:1403-1408.

22. Lee SH. Intestinal permeability regulation by tight junction: implication on inflammatory bowel diseases. Intest Res 2015; 13:11-18.

23. Ferguson LR, Huebner C, Petermann I, et al. Single nucleotide polymorphism in the tumor necrosis factor-alpha gene affects inflammatory bowel diseases risk. World J Gastroenterol 2008;14:4652-4661.

24. Thiebaut R, Kotti S, Jung C, et al. TNFSF15 polymorphisms are associated with susceptibility to inflammatory bowel disease in a new European cohort. Am J Gastroenterol 2009;104:384-391.

25. Hugot JP, Chamaillard M, Zouali H, et al. Association of NOD2 leucine-rich repeat variants with susceptibility to Crohn's disease. Nature 2001;411:599-603.

26. Fernando MM, Stevens CR, Walsh EC, et al. Defining the role of the MHC in autoimmunity: a review and pooled analysis. PLOS Genet doi: 10.1371/journal.pgen.1000024. Published online 25 April 2008.

27. McGovern DP, Jones MR, Taylor KD, et al. Fucosyltransferase 2 (FUT2) non-secretor status is associated with Crohn's disease. Hum Mol Genet 2010;19:3468-3476.

28. Barrett JC, Hansoul S, Nicolae DL, et al. Genome-wide association defines more than 30 distinct susceptibility loci for Crohn's disease. Nat Genet 2008;40:955-962.

29. Brant SR. Promises, delivery, and challenges of inflammatory bowel disease risk gene discovery. Clin Gastroenterol Hepatol 2013;11:22-26.

30. Cho JH, Brant SR. Recent insights into the genetics of inflammatory bowel disease. Gastroenterology 2011;140:1704-1712. 